\title{
A British Multi-Access System on an IBM System/360 Computer
}

\author{
Ian Pyle* \\ York, England \\ ${ }^{*}$ Corresponding author: Ian Pyle, York, England
}

\begin{abstract}
HUW was designed in 1965, to provide interactive facilities for computer users at A.E.R.E. Harwell, England. The paper explains the thinking behind the design decision underlying HUW, with their implications for users and for the performance of the system. It reflects on the consequences of these decisions for users and the establishment. HUW was retired in 1982
\end{abstract}

\section{Introduction}

Following the success of the Compatible Time-Sharing System [1], and its brilliant exploitation in Project MAC at MIT (now the MIT Computer Science and Artificial Intelligence Laboratory, CSAIL), many institutions began making similar systems. Among the British ones were a multi-access extension for the supervisor for the Titan computer at Cambridge [2], COTAN [3-4] at Culham on an English Electric KDF-9, KOS [5] at the University of Kent at Canterbury on an Elliott 4130, and HUW at Harwell on an IBM System/360 computer, the subject of this paper. Harwell Users' Workshop - HUW - was set up to provide interactive facilities for computer users at A.E.R.E Harwell. HUW was designed in 1965, following the author's sabbatical visit to Project MAC.

\section{Background}

The Atomic Energy Research Establishment (A.E.R.E.) at Harwell was the civil research arm of the United Kingdom Atomic Energy Authority (UKAEA). We were extensive computer users; in the early 1960s, much of the computing was done by sending batches of jobs for execution on computers at other sites of the UKAEA: Aldermaston and Risley, using IBM 704, 709, 7090 and 7030 (Stretch) computers [6]. With further growth in demand, the computing load at Harwell justified the installation of a new computer for the establishment. This had originally been intended to be a British computer, Atlas, but a political decision was made to locate it at the Atlas Laboratory [7].

\section{Technical Decisions}

The original design specification [8] said: "HUW (Harwell Users' Workshop) is essentially a file maintenance and message switching system in which a number of users at remote terminals can have access to a number of programs being executed concurrently by a central computer. Standard programs would exist for use from any terminal, some of which would allow the user to originate new programs to be executed either immediately or later. Bulk storage would be available for keeping files of information (data or programs), which may be accessed by programs in the computer".

In a way, the document was flying a kite, to show staff at Harwell what might be achieved by interactive working on a computer. It explicitly pointed out that "the provision of this system in whole or in part will depend on approval being obtained for the necessary installation of equipment, and on software implementation." We were already deeply involved in planning for a new computer system, and in that context, the technical decisions concerning HUW were taken. Significantly, my group leader, the late Alan Curtis, was instrumental in having the idea adopted by the Harwell directorate (see Acknowledgements, below).

\section{Main Computer and Operating System}

The decision to install an IBM System/360 followed naturally from the previous extensive experience of IBM mainframe computers, and the announcement of the overall architecture and initial models in 1964 and was not influenced by the decision to develop HUW. However, as the prospective benefits of interactive facilities became recognised, the particular model and operating system were carefully investigated. The two significant candidates were Model 65 with OS/360, option MFT (Multiprogramming with a Fixed number of Tasks) or Model 67 (announced in 1965) 
with OS/360, option TSS (Time-Sharing System). In spite of the apparent attraction of Model 67 with TSS, it was decided that the "safe" option was to select Model 65 with MFT, and write the HUW program ourselves as a permanent task running alongside users' jobs. In essence, this reflected the basic fact that the computer was being installed to run users' jobs, mostly programs written in Fortran.

\section{Interactive facilities}

The most important decision in HUW was to provide interactive facilities for users to prepare programs and data, and to examine the results, but not to interact with their own programs. This was a technically significant limitation from the more general facilities of project MAC, but avoided the necessity for extensive transfer of running programs from main store to backing store, with the consequent overhead, i.e. "Computer Time Spent Swapping"! The HUW program was permanently resident, running as a single task with many instances for the logged-on users.

\section{Terminals}

The machines used to give users their interaction with the main computer were chosen for ease of use and robustness. Most users were only familiar with punched cards, although some had seen typewriter-style machines used as operator consoles on earlier computers. The Teletype [9] was chosen as the most convenient; 50 Teletypes were installed by the end of 1967 [10], followed by the rest of the initial 160 in 1968 [11].

\section{Front-end computer}

In order to off-load the task of handling all the user terminals, which would have been a severe strain on the main computer, the design used a separate computer specifically for this purpose; it was called the Front-End Computer. IBM provided a specific unit, the IBM 2701 data adapter, to provide a parallel interface to external equipment. Several computers were considered for the front end rôle, and their performance calculated for different numbers of terminals. The principal contenders were the GEC/AEI Con Pac 4060 (because it was manufactured by a British company) and the PDP-8 (because it was familiar and simple). It turned out that there was a cross-over point on the performance graphs at about 100 terminals, and the Con Pac 4060 was cheaper for 160 terminals (the initial planned load). So, a Con Pac was ordered, with a special addition for it to interface with the IBM 2701. Unfortunately, after the IBM mainframe had been delivered and testing of HUW was starting, the suppliers of the Con Pac told us they were having difficulty making the interface to the IBM 2701. Rather than cancel the order, we decided to order a PDP-8 for testing purposes. Digital Equipment Corporation delivered the computer, we installed it, programmed it to communicate with the IBM 2701 and handle 8 Teletypes, and were able to test HUW using it before the Con Pac was eventually delivered and successfully integrated with the main frame.

\section{Performance}

As a result of these design decisions, particularly the limit on interactive facilities and the use of the front-end computer, the performance of the mainframe was satisfactorily maintained for the execution of users' jobs: the 1968 UKAEA Annual Report [11] reported only 3 percent of (IBM) computer time being used to serve the typical load of 20 to 30 logged on users.

\section{Availability}

The issue of computer availability is not directly visible to users in earlier styles of computer use (batch processing, sequential job execution), where operators deal with a computer crash by re-starting and re-running the last unfinished job. Users only see the effect of computer unavailability at a gross level, for example if a whole shift is lost. However, in contrast, an interactive system makes the availability of the service immediately visible to the user, and the availability of the IBM computer became an important characteristic after HUW came into wide use. Steps were taken to reduce the inconvenience by arranging for automatic restarts when possible: the HUW program contained extensive self-repair facilities [12].

\section{Further Development of HUW}

\section{Macros}

The most significant development of HUW after its initial installation was the extension to provide a "macro" handling facility. This allowed a user to define a file containing instructions to HUW (the same as from an ordinary terminal) and call for its execution by a single HUW command [13]. Thus, although the original design had not allowed users to interact with their own programs, this facility provided similar functionality, without the overhead. The macro facility was extensively used for a variety of special purposes, as illustrated below under "Exploitation". Unfortunately, a design error caused the availability of the mainframe to fall; this was eventually identified and corrected [12].

\section{Terminals}

Use of HUW expanded throughout the site at Harwell, and elsewhere using remote access (see below). More terminals were added, including "glass Teletypes" and some faster VDUs including some IBM terminals. The front ends were upgraded to PDP11s (developing a bridge interface to the IBM System Network Architecture) to handle these faster and more sophisticated terminals. At its peak, the system could serve about 500 users [14].

\section{Remote access}

Some of the terminal lines were connected to normal telephone lines, in order to allow users from outside the main site to access the IBM computer. Some of these lines were used by AEE Winfrith, another establishment of the UKAEA. A portable Teletype (like a heavy suitcase, connected by an acoustic coupler to a telephone) allowed occasional use from arbitrary locations. (I used it at home occasionally; my children had their first experience of a computer using it.) HUW was demonstrated at a conference in Grenoble in 1969 [15]. At that time, long- distance telephone connections had to be made by human operators, and the French operators were unaccustomed to a reply in the form of a modem's whistle rather than a human voice. However, the telephone link stayed up for the demonstration and HUW was international! 


\section{Data links}

Data links were installed to about 10 small computers in other buildings at Harwell handling experimental data. Following earlier work by Barnes [16], the communication with the remote computers was treated as though they were magnetic tape drives. This was before the invention of file transfer protocols such as FTP; as with magnetic tape, the only way of detecting a transmission error was by the checksum at the end of each record.

\section{Exploitation of HUW}

The use of HUW grew rapidly with the new IBM computer, and was generally used as the preferred way to submit jobs for execution. Subsequently, the main computer was upgraded to higher models of System/360, and the Operating System was upgraded to MVT (Multitasking with a Variable number of Tasks) with the Time-Sharing Option (TSO). After the macro facility had been added, use of HUW became even more widespread, as the following sections illustrate.

\section{Job File Creation}

The Job Control Language (JCL) of System/360 was notoriously troublesome. As far as HUW was concerned, the file of JCL commands was just another text file, to be created and edited by the user. To assist users in job creation, Curtis [17] developed a set of HUW macros that automatically created a job control file, using information supplied by the user in response to messages and prompt. Using these macros, Curtis reported that "From the terminal user's viewpoint, the effect with short ( 3 second) jobs can be almost as good as foreground execution."

\section{File handling and Numerical Analysis}

In his review of the use of HUW command macros, Curtis [18] reported that: "HUW command macros are widely used, especially to provide less sophisticated computer users with powerful, easilyused tools. Such macros often exploit the available facilities to the full, as do the macros of more advanced users. Several Divisions and groups have extensive sets of macros, representing considerable investments of effort, which would need replacing if HUW ceased to be available. An exhaustive list is impracticable; here we give a few examples:

a) A large set of macros (in some 40 macros totaling about 5000 lines of HUW macro source coding), written by M.E. Gubbins, is in use at Winfrith, mainly to provide facilities for management of Partitioned Data Sets (e.g. to select named members, or all members except named ones, of a PDS for incorporation into a source deck).

b) Two of our regular series of six courses at the Education Centre rely on HUW macros to provide students with simple facilities for working numerical examples by computer, because hand calculators could not cope with examples of adequate complexity. Although the total amount of HUW macro coding is not large (less than 1000 statements), this is because these macros process rather large files in a specially designed compact language in order to generate prompts to the student and incorporate his replies into a job file. c) A considerable number [not quantified] of system macros (names starting with \$ instead of M) exist and are widely used."

\section{Library Administration}

The Harwell library made use of HUW in their administration [19], as an early application of computers in this field. The authors reported: "The off-line computer-based loans system developed in AERE Library between $1968-70$ has been operational since 1 December 1970 . The system covers loans, discharges, reservations, borrower searches, etc. (ten types of transaction in all), for four kinds of document - reports, periodicals, books and pamphlets. The system uses two teletypewriters in the Library to produce 8-track punched paper tape which is input to the Harwell IBM $360 / 75$ computer. The principal output consists of printed loans lists, reissue and recall notices, diagnostics, error messages and statistical information. In addition to an improved control of loans, the system has already led to a saving in clerical effort; full benefits should be realized in 1971/72."

\section{Measurement}

In 1971, an experiment [20] was set up to measure the actual availability of HUW at a user's terminal. A Teletype was set up to send a stimulus to the computer every minute, and the results analyzed. Three situations were recognized: normal HUW operation (when the current time was recorded, in case this was the last of a period of availability); absence of service (when nothing of course could be done); and the start of a new period of availability (when the start time was recorded as the end of a period of inactivity, and a new period of activity started). This was done for a week in March 1971, and subsequently for a week in May 1971. The results showed that the periods of availability matched a Poisson process, with an expected time to failure of 46.5 minutes in March, which improved to 108 minutes in May. The times to repair were not distributed uniformly, in March being 16.8 minutes for short periods but 50 minutes for long periods; in May the corresponding values were 14.1 minutes for short periods, and 21.4 minutes for long periods. Checking back to the events in the operators' logs, it became clear that many short breaks had not been observed by the operators at all but had been corrected automatically within HUW. The long breaks however, sometimes needed an Initial Program Load (IPL) action by the operators, which took about 30 minutes. The availability level achieved in May (available time to unavailable time ratio 10.4 , i.e. $91.2 \%$ availability) was still considered not good enough.

\section{Messaging and text handling}

Almost incidental in the initial design, the message handling facilities in HUW were found widely useful (long before email had been invented). As well as for technical communication between users, messages were used socially and for group communication. Hance [14] reports that the messaging service was used by union activists throughout the Authority to arrange a series of half-day strikes; in reaction, the union was asked by the Harwell management to pay for this "unofficial" use of the equipment! 


\section{Review of Design Decisions}

With hindsight, it is clear that while some of the design decisions were certainly beneficial, there were others (not necessarily made consciously) that had a negative impact, and eventually led to the end of the project. The first beneficial decision was undoubtedly the restriction on user interaction which resulted in low overhead on operation of main computer. Users also appreciated the interactive facilities provided extensive use for program preparation, although not for interaction with a running program. Clearly a significant benefit was the opportunity created for administrative use, e.g. for the library loans system. Another benefit was the innovative use of computer messaging (before e-mail).

On the negative side, the design was strongly constrained to IBM System/360, its filing system and its assembly language. As a result, when Harwell eventually decided to install a computer with a different architecture, HUW could no longer be used. The limited interactive facilities for users were overcome to a considerable extent, but the advances in computer technology made them unacceptable in the long run. The decision to use Teletypes (always running at 10 characters per second) required new front-end computers to take advantage of higher-speed terminals (VDUs). This showed that the original design had been deficient.

\section{Conclusion}

HUW was in constant use at Harwell: all day, every day, from 1968 until the early 1980s, when the IBM System/360 was replaced by a Cray computer. It was not worth recoding the program for the different architecture. It had served the establishment satisfactorily for well over a decade and introduced staff to new ways of using computers. In retrospect, HUW might be considered an example of a successful computer project: one that served its purpose and satisfied its users. What were the influences that lay behind its success? In my view, the main reason was that it was home grown: a solution to a problem that was well understood by the designers, who understood their users' needs and priorities. It was made by people of the community of intended users. The "Requirements" had not been formally articulated before the design was carried out. The project was not outsourced to a separate company with commercial imperatives. Local expertise was encouraged and cherished.

\section{Acknowledgements}

The work described in this paper was initiated while the author was with the United Kingdom Atomic Energy Authority, a member of the Applied Mathematics Group, at the Atomic Energy Research Establishment, Harwell, Berkshire, England, U.K. Harwell was an ideal environment for scientific and engineering innovation, and I am extremely grateful for the opportunities given me there. The main responsibility for getting the whole project approved by the Harwell directorate was taken by my group leader, the late Alan Curtis [21]. While continuing to give me the fullest support and encouragement, he arranged for the computers and terminals to be purchased, and the cabling installed throughout the Harwell site.
After the main design had been carried out, I was transferred to work on a different project, and the work of completing the design, writing the software, testing and installation was done by Bob McLatchie [15]. He continued the development and maintenance of HUW throughout its life. I am grateful for his work in realizing the vision of the project. Furthermore, I thank Bob McLatchie for reading and commenting on an earlier draft of this paper, and for correcting several of my mistakes. I thank the UKAEA Archivist at Culham, Maya Riddle, for her work in locating the relevant documents from the Authority's archive.

\section{References}

1. Corbató FJ, etal. (1963) The Compatible Time-Sharing System: A Programmers Guide.

2. Hartley DF, Landy B and Needham RM (1968) The structure of a multiprogramming supervisor. The Computer Journal 11(3): 247-255.

3. Poole PC (1970) Developing a multi-access system online. Software: Practice and Experience 1(1): 39-50.

4. Poole PC and Lang T (1968) The development of on-line computing facilities for the KDF9 part 1; COSEC - a single on-line console. The Computer Journal 11(1): 5-11.

5. Brown PJ (1971) The Kent on-line system. Software: Practice and Experience 1(3): 269-27.

6. http://www03.ibm.com/ibm/history/exhibits/mainframe/mainframe_profiles.html.

7. Howlett J (1989) Computing at Harwell. FLAGSHIP Newsletter Issue 05 (Rutherford Appleton Laboratory, Central Computing Department).

8. Pyle IC Proposed Specification of a Computing System Using Remote Consoles. AERE M 1627 (NBS \# 6522940).

9. http://en.wikipedia.org/wiki/Teleprinter.

10. UKAEA (1967) Annual report paragraph 299.

11. UKAEA (1968) Annual report paragraph 335.

12. Pyle IC, McLatchie RCF, Grandage B (1971) A second-order bug with delayed effect. Software: Practice and Experience 1(3): 231-233.

13. Norman VJ (1970) Command Macros for HUW.AERE R 6383.

14. Nick Hance (2006) "Harwell - The Enigma Revealed". Enhance Publishing pp.196.

15. Mc Latchie RCF (1969) HUW, an interactive computer system on IBM System 360/65. SEAS XIV, Conference, Grenoble.

16. Barnes RCM (1961) High speed data transmission links as an aid to the use of major computers at a distance. AERE report $\mathrm{R} 3612$.

17. Curtis AR (1978) HUW Macros for conversational job file creation. AERE report CSS- 60 .

18. Curtis AR HUW Command Macros and their use. AERE report M 3068.

19. Wilson CWJ, Greenhalgh KR (1971) "AERE Library Computer Based Loans System- Coblos". Program: electronic library and information systems 5(2): 89-118.

20. Pyle IC (1971) Measurements of HUW availability and usage. A.E.R.E.-R.6915, Presented at the SEAS Conference, Pisa.

21. Jones I (2008) Alan Raymond Curtis (1922-2008) [in] Newsletter of the Numerical Analysis Group of the Institute of Mathematics and its Applications. 32(3): 1-3. 
(C) (P) This work is licensed under Creative To Submit Your Article Click Here: Submit Article

DOI: $10.32474 /$ CTCSA.2019.01.000120

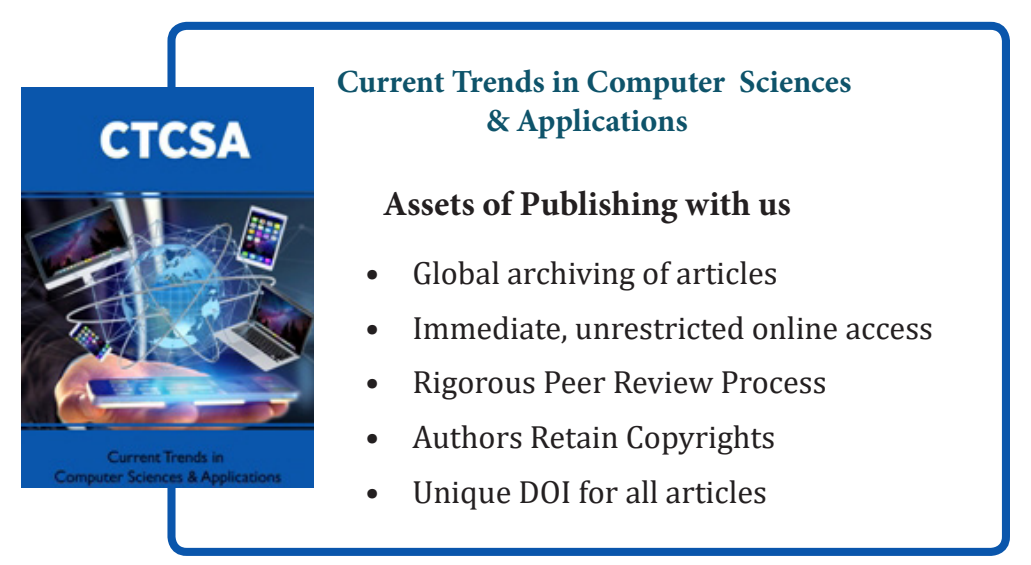

